\title{
Importância do amor-crescido (Portulaca pilosa L.) para a medicina tradicional amazônica: uma revisão bibliográfica
}

The importance of amor crescido (Portulaca pilosa L.) for traditional Amazonian medicine: a bibliographic review

Importancia del amor crescido (Portulaca pilosa L.) para la medicina tradicional amazónica: una revisión bibliográfica

Dayse Lucia Nascimento Brandão ${ }^{1,2}$, Valdicley Vieira Vale ${ }^{1,2}$, Andreza do Socorro Silva da Veiga ${ }^{1}$, Fernando Tobias Silveira ${ }^{3}$, Sandro Percário ${ }^{4}$, Márlia Regina Coelho-Ferreira ${ }^{5}$, Maria Fâni Dolabela ${ }^{1 *}$.

\section{RESUMO}

Objetivo: Identificar os usos populares de P. pilosa na região amazônica brasileira, e verificar se existem estudos que confirmem a sua atividade biológica Métodos: Trata-se de estudo qualitativo de uma revisão bibliográfica de estudos etnobotânicos realizados na região amazônica brasileira e farmacológicos. A pesquisa da literatura foi realizada nas bases de dados científicas sem restrições de data, em periódicos nacionais, internacionais, livros e trabalhos acadêmicos. Resultados: Várias alegações de uso foram constatadas, entre estes citam-se para doenças hepáticas, problemas de estômago, diarreia, como vermífugo, antimalárico, combater a queda de cabelo, cicatrizante, anti-inflamatório e analgésico. O potencial farmacológico das três últimas indicações foi comprovado. Considerações finais: Apesar da importância medicinal da espécie vegetal para Amazônia, os estudos farmacológicos ainda são escassos, sendo urgente a realização de estudos que validem seu uso para o tratamento de doenças hepáticas e gástricas, bem como sua atividade anti-inflamatória, assim $P$. pilosa apresenta-se como uma planta de grande interesse para a comunidade amazônica, bem como para comunidade científica.

Palavras-chave: Portulaca, Plantas medicinais, Etnobotânica, Etnofarmacologia, Ecossistema amazônico.

\section{ABSTRACT}

Objective: To identify the popular uses of $\mathrm{P}$. pilosa in the Brazilian Amazon region, and to verify if there are studies that confirm its biological activity. Methods: This is a qualitative study of a bibliographic review of ethnobotanical studies conducted in the Brazilian Amazon region and pharmacological studies. The literature search was performed in scientific databases without date restrictions, in national, international journals, books and academic papers. Results: Several use claims were found, among them are liver diseases, stomach problems, diarrhea such as deworming, antimalarial, combating hair loss, healing, anti-inflammatory and analgesic. The pharmacological potential of the last three indications has been proven. Final considerations: Despite the medicinal importance of the plant species for the Amazon, pharmacological studies are still scarce, and studies that validate its use for the treatment of liver and gastric diseases, as well as their anti-inflammatory activity, are urgent. $P$. pilosa presents itself as a plant of great interest to the Amazonian community as well as to the scientific community.

Keywords: Portulaca, medicinal plant, ethnobotany, ethnopharmacology, Amazonian ecosystem.

\footnotetext{
${ }^{1}$ Pós-Graduação em Inovação Farmacêutica, Universidade Federal do Pará, Belém - PA.

*E-mail: fanidolabela20@gmail.com

2 Pós-Graduação em Ciências Farmacêuticas, Universidade Federal de Minas Gerais, Belo Horizonte - MG

3 Instituto Evandro Chagas, Ananindeua - PA.

${ }^{4}$ Pós-graduação em Biodiversidade e Biotecnologia, Universidade Federal do Pará, Belém - PA.

${ }^{5}$ Pós-graduação em Biodiversidade e Biotecnologia, Museu Paraense Emilio Goeldi, Belém - PA.
}

SUBMETIDO EM: 11/2019

ACEITO EM: 12/2019

PUBLICADO EM: 3/2020 


\section{RESUMEN}

Objetivo: identificar los usos populares de la $P$. pilosa en la región amazónica brasileña, Y si como si existen estudios que confirmen su actividad biológica. Metodología: es una revisión bibliográfica los estudios etnobotánicos realizados en la región amazónica brasileña y farmacológicos. La búsqueda de literatura se realizó en las bases de datos sin restricciones de fechas, en los periódicos acionales, internacionales, libros y trabajos académicos. Resultados: se encontraron varias alegaciones del uso, la enfermedad hepática, problemas estomacales, diarrea, vermífuga, antipalúdica, combatir la caída del cabello, curación, antiinflamatoria y analgésica. Se ha demostrado el potencial farmacológico de las últimas tres indicaciones. Consideraciones finales: a pesar de la importancia medicinal de las especies de las plantas para la Amazonía, los estudios farmacológicos aún son escasos, y los estudios que validan su uso para el tratamiento de las enfermedades hepáticas y gástricas, así como su combatir la caída del cabello, son urgentes. Así, $P$. pilosa se presenta como una planta de gran interés para la comunidad amazónica, así como para la comunidad científica.

Palabras clave: Portulaca, Plantas medicinales, Etnobotánica, Etnofarmacología, Ecosistema amazónico.

\section{INTRODUÇÃO}

A história do uso de plantas com fins medicinais remonta aos primórdios das civilizações, e há quem afirme que esta história se confunde com da humanidade. Os primeiros registros de utilização de plantas medicinais são datados de 500 a. C. (antes de Cristo), no texto Chinês; e no manuscrito Egípcio "Ebers Papirus", de 1.500 a. C., em que continham 811 prescrições e 700 drogas (DUARTE MCT, 2006). Este uso se perpetuou até o século atual, sendo as plantas utilizadas em países desenvolvidos e em desenvolvimento (VEIGAJUNIOR VF, et al., 2005).

O Brasil, país em desenvolvimento, é constituído pelos biomas: a Floresta Amazônica; o Pantanal; o Cerrado de savanas e bosques; a Caatinga de florestas semi-áridas; os campos dos Pampas; e a floresta tropical pluvial da Mata Atlântica. Estes biomas variados reflete a enorme riqueza da flora e a maior biodiversidade do planeta. Além disso, há uma grande diversidade cultural no Brasil e o uso das plantas medicinais resulta de diferentes saberes construídos ao longo do tempo (PEREIRA ACS e CUNHA MGC, 2015). Na Amazônia brasileira, índios, caboclos, ribeirinhos, seringueiros, quilombolas, pescadores, pequenos produtores rurais e extrativistas são detentores de rico conhecimento sobre as plantas, sendo passado de geração em geração pela oralidade. Sabe-se ainda que a pluralidade cultural do país influenciou positivamente no uso etnobotânico de plantas medicinais, e de certa forma para o aumento da biodiversidade, haja vista a inserção de espécies exóticas na flora nacional, trazidas pelos diferentes povos que para o país vieram (VÁSQUEZ SPF, et al., 2014).

Várias espécies vegetais têm sido utilizadas a várias gerações, dentre as espécies tem-se a Portulaca pilosa L. (Portulacaceae), planta nativa não endêmica no território nacional, sendo distribuída em quatro regiões: Norte (Amazonas, Pará e Tocantins), Nordeste (Bahia, Ceará e Rio Grande do Norte) Centro-Oeste (Goiás, Mato Grosso do Sul e Mato Grosso) e Nordeste (Alagoas, Paraíba, Pernambuco e Sergipe. Nestas regiões, esta espécie é conhecida popularmente como amor- crescido, alecrim-de-São-José, flor-de-las-doce, flor-de-melodia, flor-de-seda (FERREIRA FA, 2012). A P. pilosa são atribuídas diferentes propriedades medicinais, entre elas anti-inflamatória, cicatrizante, para combater a queda de cabelo, tratamento de diarreia, problemas gástricos e antimalária (COELHO-FERREIRA MR e JARDIM MAG, 2005; SOUZA CVV, 2010; VEIGA JB e SCUDELLER VV, 2015). Porém, as alegações de uso necessitam de estudos, assim com uma vasta analise das alegações de uso está relacionada aos constituintes presente na espécie. Para a $P$. pilosa apenas foi comprovada a atividade antinociceptiva, sendo esta uma importante atividade, porém não foi observado efeito anti-inflamatório por via oral (FERREIRA FA, 2012).

Devido à importância medicinal de $P$. pilosa, esta espécie foi inserida na relação nacional de plantas de interesse para o Sistema Único de Saúde do Brasil (RENISUS). O objetivo da da criação da RENISUS foi 
orientar estudos químicos, que avaliem a segurança, eficácia da espécie vegetais para o tratamento de determinadas doenças. Desta forma, poder disponibilizar para o Sistema Único de Saúde, espécies vegetais utilizadas na medicina popular, cujo atividade e segurança seja conhecida. Este estudo objetiva identificar os usos populares de $P$. pilosa na região amazônica brasileira, bem como se existem estudos que confirmem a atividade medicinal.

\section{MÉTODOS}

Trata-se de um trabalho qualitativo, realizado através de uma revisão bibliográfica, por meio de um levantamento de publicações em periódicos nacionais e internacionais. A pesquisa da literatura foi realizada em bases indexadas de portais e periódicos (Google acadêmico e periódicos Capes) sem restrições de data. Como critérios de inclusão do estudo utilizou: ser um estudo realizado na região amazônica brasileira, o trabalho deveria relatar os resultados obtidos no estudo etnobotânico e apresentar relato de uso de Portulaca pilosa. Foram excluídos os trabalhos em duplicidade, ou que o foco não foi etnobotânico e/ou de atividade biológica. Também foram excluídos, estudos etnobotânicos realizados em outras regiões do Brasil, ou em outros países e ainda quando não estava claro o local em que se realizou o estudo. Por último, foram excluídos estudos que não estavam disponíveis, na integra, online (GALVÃO T e PEREIRA M, 2014).

No levantamento bibliográfico foram utilizados os descritores Portulaca pilosa, etnobotânica, etnofarmacologia, ecossistema Amazônico. Devido não ter limitado a região, apareceu estudos realizados em outras regiões ou países que foram excluídos. Na seleção do material, inicialmente, foram lidos os títulos e os resumos. Os artigos selecionados foram lidos integralmente. Quando estavam em consonância com o estudo, coletou-se as seguintes informações: nome dos autores, título do artigo; população estudada; local em que se residiam; forma de utilização da planta; alegações de uso; e qualquer informação considerada relevante. A falta de alguma informação não implicou na exclusão do estudo.

As informações coletadas foram organizadas em um banco de dados de e as alegações de uso foram computadas e selecionou-se as alegações mais citadas. Após esta seleção, foi realizado um novo levantamento bibliográfico que visava a identificação de estudos relacionados as alegações de usos. Nesta nova busca, todos os estudos que avaliaram a atividade alegada foram considerados, mesmo que os resultados fossem negativos. No caso do efeito cicatrizante, considerou-se ainda que, a ferida de difícil cicatrização pode estar relacionada a infecção por leishmaniose e por isso, considerou-se estudo relacionado ao efeito leishmanicida de $P$. pilosa. Vale ressaltar que, estudos que relataram a atividade de outras espécies pertencentes ao gênero não foram considerados, bem como trabalhos que avaliaram, apenas, o potencial dos metabolitos secundários presentes na $P$. pilosa.

Em relação à pesquisa sobre estudos etnobotânicos de $P$. pilosa foram encontrados 355 estudos na base de dados do Google Acadêmico e apenas 2 artigos no portal periódicos Capes, o total de produtos encontrados foi 357. Após a exclusão de estudos em duplicidade, que não estavam disponíveis na integra na plataforma online, ou que o foco não era a etnobotânica, restaram apenas 25 estudos, sendo 24 artigos científicos publicados em diferentes periódicos e 1 dissertação de mestrado da Universidade Federal do Pará.

\section{RESULTADOS E DISCUSSÃO}

\section{Portulaca pilosa e seu uso medicinal}

A P. pilosa é muito utilizada pelas comunidades amazônicas com fins medicinais e cosméticas. Na Tabela 1, observa-se que comunidades ribeirinhas, quilombolas, residentes em diferentes estados da Amazônia legal utilizam esta espécie com diferentes objetivos terapêuticos, sendo as folhas a parte da planta mais utilizada. Ressalta-se que esta espécie é herbácea com folhas carnosas e flores vermelhas em cachos terminais, de fácil cultivo, isto é, pode ser produzida em hortas caseiras. O estudo de Flor e Barbosa chama a atenção para a ampla utilização de espécies herbáceas nos sistemas de cura populares (FLOR ASSO e BARBOSA WLR, 2015). 
Além disso, Amazônia, por possuir vasta área territorial, acesso dificultado via terrestre, pois parte das comunidades apresentam como o único acesso à via fluvial ou aérea, propicia o uso de plantas medicinais como único recurso para o tratamento das enfermidades. Alguns estudos etnobotânicos relatam a importância medicinal desta espécie para a comunidade, merecendo destaque o estudo realizado em Barão de Igarapé Miri, PA, Brasil, onde a $P$. pilosa é citada como uma das 10 espécies medicinais mais importante para a comunidade (PINTO LN, 2008). Entretanto, este e outros estudos não descrevem as alegações de uso popular da espécie, e também a forma de utilização não foi descrita (Tabela 1).

Neste trabalho verificou-se um total de 25 estudos etnobotânicos com a espécie e várias alugações de uso foram relatadas os seguintes: estomáquica, diurética, cicatrizante, analgésica, doenças hepáticas, malária, ulceras, queimaduras, erisipelas, depurativo sanguíneo, diarreia, desinterias, cólicas, hemoptises, nefrites, vermífugo, icterícia, queda de cabelo, doer de estômago, fraturas e luxação muscular. Quanto às partes utilizadas da planta para o preparo dos remédios observou-se que $56 \%$ dos estudos não foram relatadas as partes utilizadas. A folha é a parte do vegetal mais utilizada (32\%) nos artigos seguida do decocto de toda planta $(8 \%)$ e somente $4 \%$ utilizou raminhos. A principal forma de preparo dos remédios com a planta foi através do chá $(28 \%)$. As preparações caseiras foram realizadas também na forma de compressa tópica (4\%), sumo e garrafadas (4\%), infusão (4\%), suco (4\%) e remédio caseiro (4\%), sendo que em $56 \%$ dos estudos não foram relatados o modo de preparo. Somente em um estudo houve o preparo da planta junto a outro vegetal para aumentar a eficácia do tratamento (Tabela 1).

As diferentes alegações de uso para uma mesma espécie, pode ser resultado da junção dos conhecimentos dos índios, caboclos, ribeirinhos, seringueiros, quilombolas, pescadores, pequenos produtores rurais e extrativistas (VÁSQUEZ SPF, et al., 2014). Entretanto, a maioria destes "saberes" populares carecem de estudos de validação para a atividade, bem como para sua segurança. A consequência da não realização destes estudos é que muitas substâncias, presentes nas espécies medicinais e com elevada atividade, não são identificadas e vários candidatos a fármacos são perdidos. Em relação aos investimentos nas plantas medicinais amazônicas, a indústria farmacêutica investe pouco e o governo brasileiro poderia ter mais editais, financiando pesquisas com este foco. 
Tabela 1 - Alegações de uso popular de P. pilosa na Amazônia brasileira

\begin{tabular}{|c|c|c|c|c|}
\hline Local de realização do estudo & Alegações de uso popular & Parte usada & $\begin{array}{c}\text { Modo de } \\
\text { administração }\end{array}$ & Referência \\
\hline Porto Velho, RO, Brasil & Febre & NR & NR & NEVES ES, 1980 \\
\hline Região Norte & $\begin{array}{l}\text { Estomáquica, diurética, cicatrizante, } \\
\text { analgésica, em casos de doenças } \\
\text { hepáticas, malária, úlceras }\end{array}$ & NR & NR & SILVA FA, et al., 1998 \\
\hline Bacia amazônica, Brasil & $\begin{array}{l}\text { Diarreia, disenteria, cólica, nas } \\
\text { hemoptises, nefrites e como vermífugo; } \\
\text { feridas, eritemas e icterícia }\end{array}$ & NR & NR & REVILLA J, 2002 \\
\hline $\begin{array}{l}\text { Comunidade Pesqueira de Marudá, } \\
\text { PA, Brasil }\end{array}$ & NR & NR & NR & $\begin{array}{l}\text { COELHO-FERREIRA MR e } \\
\text { SILVA MFF, } 2005\end{array}$ \\
\hline Santa Barbara do Pará, PA·, Brasil & $\begin{array}{l}\text { Inflamação, Baque, estômago, fígado, } \\
\text { rim, }\end{array}$ & Folhas & $\begin{array}{l}\text { Sumo, chá } \cdot \text {, garrafada } \\
\text { de folhas }\end{array}$ & $\begin{array}{l}\text { COELHO-FERREIRA MR e } \\
\text { JARDIM MAG., } 2005\end{array}$ \\
\hline Barão de Igarapé Miri, PA, Brasil & NR & NR & NR & PINTO LN, 2008 \\
\hline $\begin{array}{c}\text { Comunidade São João (SJ) rio Negro } \\
\text { e lago Tupé, AM, Brasil }\end{array}$ & NR & NR & NR & SCUDELLER VV, et al., 2009 \\
\hline $\begin{array}{l}\text { Agricultores familiares de Manacapuru } \\
\qquad(\mathrm{AM})\end{array}$ & NR & NR & NR & COSTA JR e MITJA D, 2010 \\
\hline $\begin{array}{l}\text { Comunidades ribeirinhas, Manaus, } \\
\text { AM, Brasil }\end{array}$ & $\begin{array}{l}\text { Inflamação, fígado, ferimentos e queda de } \\
\text { cabelo }\end{array}$ & NR & & SOUZA CVV, 2010 \\
\hline Comunidade São João do Tupé, AM & Malária & NR & NR & $\begin{array}{l}\text { VEIGA JB e SCUDELLER VV, } \\
2015\end{array}$ \\
\hline $\begin{array}{l}\text { Comunidades quilombolas do oeste do } \\
\text { Pará }\end{array}$ & NR & NR & NR & SILVA TVA, et al., 2011 \\
\hline Barcelos, Amazonas & Fígado & Folha & Infusão & TOMCHINSKY B, 2013 \\
\hline Manacapuru, AM, Brasil & $\begin{array}{c}\text { Inflamação, fígado, queda de cabelo, } \\
\text { próstata }\end{array}$ & Folha & $\begin{array}{l}\text { Chá, banho, sumo- } \\
\text { Interno e externo }\end{array}$ & VÁSQUEZ SPF, et al., 2014 \\
\hline Marudá, PA, Brasil & Anti-inflamatório & NR & NR & $\begin{array}{l}\text { FLOR ASSO e BARBOSA } \\
\text { WLR, } 2017\end{array}$ \\
\hline
\end{tabular}

$\overline{\text { REAS/EJCH | Vol.12(3)| e2371 | DOI: https://doi.org/10.25248/reas.e2371.2020 Página } \mathbf{5} \text { de } \mathbf{1 1}}$ 


\section{Revista Eletrônica Acervo Saúde / Electronic Journal Collection Health ISSN 2178-2091}

\begin{tabular}{|c|c|c|c|c|}
\hline Vale do Juruena, MT, Brasil & Lesões dermatológicos e úlcera gástricas & NR & NR & BIESKI IGC, et al., 2015 \\
\hline Rio Branco-Acre e Sul AM- Brasil & Malária e fígado & $\begin{array}{l}\text { Decocto de } \\
\text { toda planta }\end{array}$ & Chá & FERREIRA AB, et al., 2015 \\
\hline Abaetetuba, PA, Brasil & Dor no estômago & $\begin{array}{l}\text { Decocto de } \\
\text { toda planta }\end{array}$ & Chá & MOURA PHB, et al., 2016 \\
\hline Abaetetuba, PA, Brasil & NR & NR & & FERREIRA LB, et al., 2016 \\
\hline Uruará, Pará, Brasil & Vermes; estômago. & Raminhos & Suco & CAJAIBA CA, et al., 2016 \\
\hline $\begin{array}{l}\text { Comunidade ribeirinha Santa Luzia em } \\
\text { Manacapuru-AM }\end{array}$ & NR & NR & NR & DAMASCENO JO, 2017 \\
\hline $\begin{array}{c}\text { Casa de Tupinambá, Tracuateua, PA, } \\
\text { Brasil }\end{array}$ & NR & Folha & Remédio caseiro & OLIVEIRA NJG 2017 \\
\hline Ananindeua e Marituba/PA, Brasil & NR & Folha & NR & SANTOS JJF, et al.,2018 \\
\hline Mercado de Manaus, AM, Brasil & $\begin{array}{l}\text { Estomáquica, diurética, cicatrizante, } \\
\text { analgésica, em casos de doenças } \\
\text { hepáticas, malária, úlceras, diarreia, } \\
\text { disenteria, cólica, nas hemoptises, } \\
\text { nefrites e vermífugo }\end{array}$ & Folhas & Chá & PINHEIRO KTJS, 2018 \\
\hline Vale do rio Araguari, Amapá, Brasil. & Músculos puxados, fraturas & Folhas & Chá & MORAES LLC, et al., 2019 \\
\hline $\begin{array}{l}\text { Comunidade quilombolas de Tauerá- } \\
\text { Açú, Abaetetuba, Pará, Brasil. }\end{array}$ & $\begin{array}{l}\text { Inflamação de lesões }(A) \text {, Dor }(B) \text {, } \\
\text { Problema no estômago }(C)\end{array}$ & Folhas & $\begin{array}{l}\text { A) In natura: bate as } \\
\text { folhas e por sobre } \\
\text { lesão ou Unguento (B) } \\
\text { Chá:coloca para ferver } \\
\text { as folhas do amor- } \\
\text { crescido com erva- } \\
\text { cidreira e água; (C) } \\
\text { Chá: ferve a folha com } \\
\text { amor crescido e } \\
\text { sicuriju,. }\end{array}$ & $\begin{array}{l}\text { PEREIRA MGSG e COELHO- } \\
\text { FERREIRA M, } 2017\end{array}$ \\
\hline
\end{tabular}

Legenda: NR: não relatada. Fonte: Brandão DLN, et al., 2019

REAS/EJCH | Vol.12(3)| e2371 | DOI: https://doi.org/10.25248/reas.e2371.2020 Página $\mathbf{6}$ de 11 
Um fato merece ser destacado, isto é, na região norte do Brasil é comum o uso da $P$. pilosa para a queda de cabelos e como fortalecedor de crescimento capilar, sendo utilizados xampus (MORS BW, et al., 2000). O mercado do Ver-o-Peso, situado em Belém do Pará e farmácias de manipulação comercializam formulações a base de xampus, cremes e loções contendo a planta. O fabricante Artesanato Juruá, também situado na cidade de Belém PA, relata que o uso do xampu de Amor-crescido contribui para o fortalecimento raízes do cabelo, estimulando seu crescimento, conferindo brilho e maciez ao cabelo, sem falar nas várias formulações caseiras que são vendas nas feiras (BARATA LES, et al., 2009).

Diferentes alegações de uso da $P$. pilosa foram encontradas, sendo que as mais citadas foram para o tratamento de doenças hepáticas, estomáquicas e outras gástricas, cicatrizante, antimalárico, tratamento de inflamações, diarreias ou vermífugo, ainda para tratar a queda de cabelo. A Tabela 2 apresenta as principais alegações de uso.

Tabela 2 - Principais alegações de uso popular de $P$. pilosa na de diferentes cidades pertencentes a amazônica brasileira.

\begin{tabular}{lc}
\hline Alegações & Número de citações \\
\hline Doenças hepáticos & 8 \\
Estomáquica e outras doenças gástricas & 8 \\
Cicatrizante & 3 \\
Antimalárico e febre & 4 \\
Tratamento de inflamações & 5 \\
Diarreias e vermífugo & 3 \\
Queda de cabelo & 2 \\
Analgésica & 2 \\
\hline
\end{tabular}

Fonte: Brandão DLN, et al., 2019

\section{Alegações de uso e atividade biológicas}

Apesar do extenso uso popular, ainda existe carências de estudos que avaliaram a atividade sobre as doenças hepáticas, estomáquica e outras doenças gástricas. A consequência da escassez de estudo pode impactar na obtenção de novo fitoterápico, bem como limitar o avanço da indústria farmacêutica para áreas pouco exploradas (ANTONIO GD, et al., 2013). Assim, estudos que que visem a pesquisa sobre essas atividades biológicas se tornam urgentes. Entre as atividades biológicas já relatadas na literatura para $P$. pilosa, destacam-se:

Atividade tópica cicatrizante do gel de $P$. pilosa e do extrato de propilenoglicol de $P$. pilosa $(150 \mathrm{mg} / \mathrm{kg}$; miligrama por quilograma) em lesões cirúrgicas de ratos wistar foi avaliado. O extrato modulou a resposta inflamatória do tecido, estimulou a angiogênese e a proliferação de fibroblastos. Nos grupos tratados com $P$. pilosa a cicatrização foi melhor que o controle negativo, sendo observado um padrão de organização da epiderme e derme melhor, obtendo um leve processo inflamatório, com proliferação de fibroblastos e aumento da formação de fibras de colágeno. A atividade cicatrizante da espécie, provavelmente, está relacionada ao ácido gálico. Estes resultados sugerem que a atividade tópica cicatrizante se deve a atividade anti-inflamatória (ALVES-BARROS AS, et al., 2017). A atividade anti-inflamatória em modelos de carragenina e o extrato hidroetanólico de partes aéreas de $P$. pilosa nas concentrações de 400 e $600 \mathrm{mg} / \mathrm{kg}$ (via oral) não inibiu a formação de edema de modo considerável em ambos os testes (FERREIRA FA, 2012). Logo, os resultados da atividade anti-inflamatória de $P$. pilosa são contraditórios, isto é, quando administrados por via tópica, observou redução do processo inflamatório, enquanto que, por via oral não houve alteração do processo inflamatório. 
Apesar de não ter sido observado efeito anti-inflamatório por via oral, foi observada atividade antinociceptiva significativa. No teste da formalina, o tratamento com extrato hidroetanólico nas concentrações 200,400 e $600 \mathrm{mg} / \mathrm{kg}$ (via oral) reduziu de modo significativo o tempo de lambida nas fases neurogênica ( $1^{\mathrm{a}}$ fase) e inflamatória ( $2^{a}$ fase). A administração prévia de naloxona, L-NAME, azul de metileno ou glibenclamida reverteu, significativamente, o efeito do extrato $(600 \mathrm{mg} / \mathrm{kg}$, via oral.) nas duas fases do teste da formalina. $O$ pré-tratamento com o L-NAME e com azul de metileno reverteu o efeito do extrato hidroetanólico duas fases do teste da formalina de maneira significativa.

Os resultados estudo demostraram que o extrato quando administrado por via oral apresentou baixa toxicidade e sua atividade antinociceptiva observada na fase neurogênica pode estar relacionada as interações com receptores opióides e ativação da via NO/GCs/GMPc/ KATP. Já a atividade antinociceptiva observada na fase inflamatória parece não depender de inibição da via bioquímica fosfolipase A2/ciclooxigenases, mas de interações periféricas com receptores opióides e com a via NO/GCs/GMPc/KATP (FERREIRA FA, 2012).

Na região amazônica, muitas vezes, a ferida está relacionada a infecção por Leishmania. Visando verificar se a $P$. pilosa possui atividade leishmanicida, e por consequente, pode contribuir para a cicatrização de feridas relacionadas a este parasito. O presente grupo avaliou a atividade contra formas promastigota e amastigota de $L$. amazonensis do extrato etanólico obtido das partes áreas da planta. Infelizmente, o extrato não foi ativo na forma promastigota e nem interferiu no índice de infecção dos macrófagos (VEIGA ASV, 2013).

O estudo realizado contra o Plasmodium in vitro foi observado que o extrato etanólico obtido das partes áreas de $P$. pilosa foi ativo apresentado baixa citotoxicidade para macrófago e observando-se elevada seletividade. Estudos adicionais para atividade antimalárica, utilizando modelos in vivo, precisam ser realizados, também a identificação do composto envolvido na atividade é importante (BRANDÃO DLN, 2012).

A diarreia pode resultar de um processo infeccioso bacterianos, em especial pela Salmonela e E. coli. Então, foi avaliada a atividade antibacteriana do extrato etanólico obtido das partes aéreas pelo presente grupo. As bactérias do gênero Salmonella são consideradas a principal causa de doença transmitida por alimentos. Sendo geralmente transmitidas para os humanos por meio do consumo de alimentos de origem animal contaminados carne, aves, ovos e leite (FORTES TP, et al., 2012).

A Escherichia coli colonizam o intestino constituindo parte de sua microbiota entérica. Sendo que a maioria das cepas não são patogênicas, algumas estirpes são associadas a várias patologias humanas e animais causam infecções intestinais (enterite, diarreia e disenteria) por adesão e lesão do epitélio intestinal ou, ainda, por ação local de toxinas enterotoxina (KALITA HU e TORRES AG, 2014).

O presente grupo avaliou a atividade antibacteriana do extrato etanólico obtido das partes aéreas de $P$. pilosa, não sendo observado efeito inibitório promissor em E. coli e Salmonela sp. Logo, a atribuição da atividade antidiarreica pode estar relacionada a ligação a receptores opióides, que ao serem ativados podem promover a redução do peristaltismo. Entretanto, estudos precisam ser realizados para confirmar esta hipótese de que a atividade está relacionada a redução do peristaltismo (FERREIRA FA, 2012).

A Tabela 3 relaciona os estudos farmacológicos realizados para algumas alegações de uso, fica claro a necessidade de estudos para as alegações de tratamento de doenças hepática, estomáquica e doenças gástricas, bem como vermífugo. Os estudos já existentes validam o uso tópico como cicatrizante, e demonstram o potencial analgésico e antimalárico da espécie. Em relação a alegação de uso e a comprovação da atividade biológica observou-se que houve atividade biológica comprovada para cicatrizante (11,11\%), antimalárico (11,11\%), tratamento da inflamação (11,11\%) e analgésica $(11,11 \%)$.

Porém, para 55,55\% das alegações populares ainda faltam estudos que comprovem a atividade biológica. Quando correlacionamos a alegação de uso e a atividade farmacológica comprovada observou-se a que as alegações de uso popular com maior destaque (Tabela 2) ainda não tiveram a sua atividade biológica comprovada farmacologicamente (doenças hepáticas, estomáquico ou doenças gástricas, vermífugo e queda de cabelo). 
Tabela 3 - Alegações uso popular e resultados dos estudos realizados

\begin{tabular}{cc}
\hline Alegações & Estudos farmacológicos \\
\hline Doenças hepáticos & Não foi encontrado estudo \\
Estomáquica e outras doenças gástricas & Não foi encontrado estudo \\
Cicatrizante & $\begin{array}{c}\text { Via tópica: atividade relacionado a } \\
\text { atividade anti-inflamatória }\end{array}$ \\
Antimalárico & Ativo \\
Tratamento de inflamações & VO - não foi ativo \\
Tiarreias & Tópica: ativo \\
Vermífugo & Não foi encontrado estudo \\
Queda de cabelo & Não foi encontrado estudo \\
Analgésica & Ativo
\end{tabular}

Fonte: Brandão DLN, et al., 2019

A planta em estudo tem baixo nível de convergência entre as informações populares e o uso farmacológico comprovado, o que sugere a necessidade de ampliação dos estudos, porém estudo etnobotânico, servirá de um norteador para os testes farmacológicos, os quais irão possivelmente comprovar, ou não, a verdadeira eficácia do vegetal. Outra questão que merece a atenção é a questão da segurança do uso cosmético e medicinal da espécie, pois existe uma lacuna de conhecimento em relação a genotoxicidade, mutagenicidade, carcinogenicidade, toxicidade in vivo, segurança do uso em gestantes, lactantes, crianças e idosos. Devido sua importância para a região amazônica, torna-se urgente a realizam destes estudos que visem validar o uso e assegurar a segurança.

Ao se analisar a importância da espécie para a Amazônia e o número de estudos já realizados com a $P$. pilosa, percebe-se há descompasso, pois poucos estudos foram realizados, sendo necessários estudos que visem comprovar o uso popular da espécie, assim como ensaios de toxicidade para validar seu uso com segurança (FERREIRA FA, 2012). Partindo deste pressuposto, cabe uma indagação o que significaria para a região a validação do uso medicinal de $P$. pilosa? Sabe-se que na região amazônica seu uso é bastante difundido, conforme ficou aqui visualizado, mas com a efetivação desses conhecimentos, e com o devido processo de validação, haveria uma ampliação de sua demanda de uso para as outras regiões brasileiras e até outros países.

O aumento de consumo poderia contribuir para a geração de renda das comunidades amazônicas, principalmente em cooperativas de plantio e colheita, haja vista a planta ser de fácil cultivo. Também, poderia ser um catalizador para o investimento em laboratórios para processamento de material vegetal e desenvolvimento de fitoterápicos ou produtos de beleza, podendo contribuir para o desenvolvimento local. Deste modo, a $P$. pilosa, além da importância medicinal, poderia assumir uma maior importância econômica para a região Amazônica.

\section{CONSIDERAÇÕES FINAIS}

A importância medicinal da $P$. pilosa para Amazônia é enorme, em especial para as comunidades ribeirinhas, quilombolas, indígenas, pequenos municípios, que são detentores dos saberes populares sobre as plantas medicinais, onde acesso ao medicamento industrializado é limitado, sendo também dificultado pelo difícil acesso aos serviços de saúde. Um problema em relação a $P$. pilosa é a falta de estudos que comprovem suas alegações de usos medicinais e estabeleça a sua forma segura de uso para todas as faixas etárias, 
assim como para pessoas com doenças crônicas. Além da comprovação cientifica das alegações de uso popular. Ainda precisa ser ressaltado que a estruturação de arranjos produtivos locais, onde a $P$. pilosa poderia ser cultivada, e onde o material vegetal poderia ser processado e comercializado na forma de droga vegetal, medicamentos ou cosméticos, como por exemplo xampoos, que poderá gerar renda para as comunidades e contribuir para a melhor qualidade de vida deles.

\section{AGRADECIMENTOS E FINANCIAMENTO}

Os autores agradecem à Capes através da concessão de bolsas de estudo. Este estudo foi financiado em parte pela Coordenação de Aperfeiçoamento de Pessoal de Nível Superior - Brasil (CAPES) - Código Financeiro 001.

\section{REFERÊNCIAS}

1. ALVES-BARROS AS, et al. Study of the non-clinical healing activities of the extract and gel of Portulaca pilosa L. in skin wounds in wistar rats: A preliminary study. Biomed Pharmacother, 2017, 96:182-190.

2. ANTONIO GD, et al. Contribuições das plantas medicinais para o cuidado e a promoção da saúde na atenção primária. Inteface, 2013, 17(46): 615-633.

3. BARATA LES, et al. Plantas Medicinais Brasileiras. II. Portulaca pilosa L. (Amor-crescido). Revista Fitos, 2009, 4(1): $126-128$.

4. BIESKI IGC, et al. Ethnobotanical study of medicinal plants by population of Valley of Juruena Region, Legal Amazon, Mato Grosso, Brazil. J Ethnopharmacol, 2015, 173: 383-423.

5. BRANDÃO DLN. Portulaca pilosa L. e Geissosperma vellosii. Estudos botânicos, farmacognósticos, fitoquímicos e atividades biológicas. Dissertação (Mestrado em Ciências Farmacêuticas) i Instituto Ciências da Saúde. Universidade Federal do Pará, Belém-PA, 2012; 177 p.

6. CAJAIBA RL, et al. Levantamento etnobotânico de plantas medicinais comercializadas no município de Uruará, Pará, Brasil. Biotemas, 2016, 29(1): 115-131.

7. COELHO-FERREIRA MR, JARDIM MAG. Algumas espécies medicinais usadas pelos moradores da ilha de Algodoal, Maiandeua, município de Maracanã,Pará. Bol Mus Para Emilio Goeldi, 2005, 1(2): 45-51.

8. COELHO-FERREIRA MR, SILVA MFF. A fitofarmacopéia da Comunidade Pesqueira de Marudá, Litoral Paraense. Bol Mus Para Emilio Goeldi, 2005, 1(2): 31-43.

9. COSTA JR, MITJA D. Uso dos recursos vegetais por agricultores familiares de Manacapuru (AM). Acta Amazon, 2010, 40: 49-58.

10. DAMASCENO JO. O uso das plantas e a cultura material e imaterial dos moradores da comunidade ribeirinha Santa Luzia em Manacapuru-am. TCC (Bacharelado em Arqueologia) - Universidade do Estado do Amazonas. Manacapuru, AM, 2017; 46 p.

11. DUARTE MCT. Atividade antimicrobiana de plantas Medicinais e Aromáticas utilizadas no Brasil. Multiciência, 2006, 7.

12. FERREIRA AB, et al. Plants Used to Treat Malaria in the Regions of Rio Branco-Acre State and Southern Amazonas State - Brazil. International Journal of Phytocosmetics and Natural Ingredients, 2015.2: 9.

13. FERREIRA FA. Avaliação das atividades antinociceptiva e anti-inflamatória do extrato hidroetanólico de partes aéreas de Portulaca pilosa L. (Portulacaceae). Dissertação (Mestrado em Ciências Farmacêutica) - Instituto Ciências da Saúde. Universidade Federal do Pará, Belém, 2012; 121 p.

14. FERREIRA LB, et al. Etnobotânica das Plantas Medicinais Cultivadas nos Quintais do Bairro de Algodoal em Abaetetuba/PA. Fitos, 2016, 10: 220-372.

15. FLOR ASSO, BARBOSA WLR. Sabedoria popular no uso de plantas medicinais pelos moradores do bairro do sossego no distrito de Marudá - PA. Brazilian journal of medicinal plants, 2015, 17: 757-768.

16. FORTES TP, et al. Ilhas de patogenicidade de Salmonella enterica: uma revisão. Salmonella enterica pathogenicity islands: a review. Rev Inst Adolfo Lutz, 2012, 71(2): 219-227.

17. GALVÃO T, PEREIRA M. Revisões sistemáticas da literatura: passos para sua elaboração. Epidemiol. Serv. Saúde, 2014, 23: 183-184.

18. KALITA A, TORRES AG. Recent advances in adherence and invasion of pathogenic Escherichia coli. Curr Opin Infect Dis, 2014, 27(5): 459-464.

19. MORAES LLC, et al. Ethno-knowledge of medicinal plants in a community in the eastern Amazon. Revista de Ciências Agrárias, 2019, 42: 565-573. 
20. MORS BW, et al. Medicinal Plants of Brazil. Michigan, USA: Reference Publicaations, Inc, 2000, 501 p.

21. MOURA PHB, et al. Etnobotânica de chás terapêuticos em Rio Urubueua de Fátima, Abaetetuba - Pará, Brasil. Biotemas, 2016, 29: 77-88.

22. NEVES ES. Introdução ao Levantamento da Flora Medicinal de Rondônia. Porto Velho: Secretaria de Ciência Tecnologia/Secretaria de Saúde, 1980, 285 p.

23. OLIVEIRA NJG. Uma abordagem etnobotânica na tenda de umbanda casa de Tupinambá, Tracuateua-PA, TCC (Bacharelado em Ciências Biológicas) - Universidade Federal Rural da Amazônia, Capanema, PA, 2017 ; 106 p.

24. PEREIRA ACS, CUNHA MGC. Medicina popular e saberes tradicionais sobre as propriedades medicinais da flora cerradeira. Hygeia, 2015, 11(21): $126-137$.

25. PEREIRA MGSG, COELHO-FERREIRA M. Uso e diversidade de plantas medicinais em uma comunidade quilombola na Amazônia Oriental, Abaetetuba, Pará. Biota Amazônia, 2017, 7(3): 57-68.

26. PINHEIRO KTJS. Espécies de uso medicinal comercializadas em duas feiras de Manaus-AM. TCC (Tecnólogo em Agroecologia) - Instituto Federal de Educação, Ciência e Tecnologia do Amazonas, Manaus - AM, 2018,43 p.

27. PINTO LN. Plantas medicinais utilizadas por comunidades do municipio de Igarapé- Miri, Pará- Etnofármacia do municipio de Igarapé- Miri- Pa. Dissertação (Mestrado em Ciências Farmacêutica) - Instituto de Ciências da Saúde, Universidade Federal do Pará, Belém, 2008; 98 p.

28. REVILLA, J. Plantas Úteis da Bacia Amazônica. Manaus, AM: SEBRAE-AM/INPA; 2002; .532 p.

29. SANTOS JJF, et al. Etnobotânica de plantas medicinais em mercados públicos da Região Metropolitana de Belém do Pará, Brasil. Biota Amazônia, 2018, 8(1): 1-9.

30. SCUDELLER VV, et al. Etnoconhecimento de plantas de uso medicinal nas comunidades São João do Tupé e Central (Reserva de Desenvolvimento Sustentável do Tupé). In: SILVA ES. Biotupé: Meio Físico, Diversidade Biológica e Sociocultural do Baixo Rio Negro, Amazônia Central. 2 V. Manaus, AM: UEA Edições; 2009; 206 p.

31. SILVA FA, et al. Obtenção e caracterização de extratos de Portulaca pilosa (Amor-crescido). XV Simpósio de Plantas Medicinais do Brasil, Águas de Lindóia: Programa e Resumos, 1998, p 185.

32. SILVA TVA, et al. Estudo etnobotânico em comunidades quilombolas do oeste do Pará. Anais do VII Congresso de Ciência e Tecnologia da Amazônia e XI Salão de Pesquisa e Iniciação Cientifica do CEULS/ULBRA Santarém Organização dos Anais: Izabel Alcina Soares Evangelista Editoração Eletrônica: Igo Tárcio Ramos de Menezes, 2011, p.124-128.

33. SOUZA CCV. Etnobotânica de quintais em 3 comunidades ribeirinhas na Amazônica Central. Manaus, AM: INPA/ UFAM; 2010, 91 p.

34. TOMCHINSKY B. Etnobotânica de plantas antimaláricas em Barcelos, Amazonas. Dissertação (Mestrado em Ciências Agronômicas) - Universidade Estadual Paulista, Faculdade de Ciências Agronômicas, Botucatu, 2013, 189 p

35. VÁSQUEZ SPF, et al. Etnobotânica de plantas medicinais em comunidades ribeirinhas do Município de Manacapuru, Amazonas, Brasil. Acta Amazônica, 2014, 44(4): 457-472

36. VEIGA ASV. Atividade antileismania de plantas da Amazônia. Dissertação (Mestrado em Ciências Farmacêuticas) Instituto Ciências da Saúde. Universidade Federal do Pará, Belém-PA, 2013; 168 p.

37. VEIGA JB, SCUDELLER VV. Etnobotânica e medicina popular no tratamento de malária e males associados na comunidade ribeirinha Julião - baixo Rio Negro (Amazônia Central). Rev. Bras plantas med, 2015, 17(4): $737-747$.

38. VEIGA- JUNIOR VF, et al. Plantas medicinais: cura segura? Quim Nova, 2005, 28(3): 519-528. 